\title{
Recent Flow Results in d+Au Collisions from Beam Energy Scan at RHIC-PHENIX
}

\author{
Shinlchi Esumi* for the PHENIX collaboration ${ }^{\dagger}$ \\ University of Tsukuba, Tomonaga Center for the History of the Universe (TCHoU) ${ }^{\ddagger}$ \\ E-mail: esumi.shinichi.gneu.tsukuba.ac.jp
}

Recent results from PHENIX experiment are presented especially on collective flow in small systems $\mathrm{p}+\mathrm{Au}, \mathrm{d}+\mathrm{Au}$ and ${ }^{3} \mathrm{He}+\mathrm{Au}$ collisions at $\sqrt{s_{N N}}=200 \mathrm{GeV}$ as well as in beam energy scan from $19.6 \mathrm{GeV}, 39 \mathrm{GeV}, 62.4 \mathrm{GeV}$ up to $200 \mathrm{GeV}$ d+Au collisions. The results include correlations between forward and backward $\eta$ regions from -3.9 to 3.9 with $\eta$-gap from 0.65 up to 6.2 units. Significant long range and near-side azimuthal correlation have been observed even with the largest $\eta$-gap of 6.2 in central d+Au collisions at $200 \mathrm{GeV}$ and this signal has found to be reduced at lower beam energies. The extracted elliptic event anisotropy parameter $v_{2}$ and the particle species dependence can be interpreted as a signal of collective and elliptic expansion, which is originally driven by the initially small and anisotropic geometry of fluctuating participant density distribution. A similar but increasing collective and elliptic expansion with system size is observed when comparing the central $\mathrm{p}+\mathrm{Au}, \mathrm{d}+\mathrm{Au}$ and ${ }^{3} \mathrm{He}+\mathrm{Au}$ collisions. The full interpretation of these results would require more complete centrality and rapidity dependent studies yet to come in near future. A new way of extracting the small flow signal from relatively large non-flow background is also discussed in order to observe any small change of the azimuthal correlation function with a reference fitting method which would be suitable especially for multiplicity dependent flow analysis in small system.

Critical Point and Onset of Deconfinement - CPOD2017

7-11 August, 2017, The Wang Center, Stony Brook University, Stony Brook, NY

\footnotetext{
* Speaker.

${ }^{\dagger}$ RHIC accelerator at Brookhaven National Laboratory (BNL), PHENIX collaboration

${ }^{\ddagger}$ former : Center for Integrated Research in Fundamental Science and Engineering (CiRfSE)
} 


\section{1. two particle correlation functions for flow measurement}

High energy proton-proton collisions at LHC has provided a new possibility to study hightemperature and high-density state of matter especially at high multiplicity environment even in

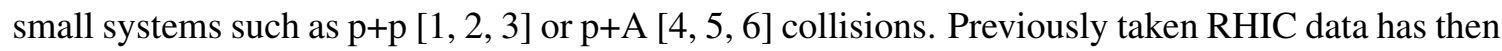
be reanalyzed to measure the azimuthal anisotropy in order to look for elliptic flow/collectivity in $\mathrm{d}+\mathrm{Au}$ collisions at RHIC energy. The long range azimuthal correlation which is known as ridge-like near-side correlation has seen in RHIC data and interpreted as elliptic collectivity in small system which is originated from the initially fluctuating density profile of participant geometry. Therefore RHIC physicists have decided to further continue studying various different small collision systems such as $\mathrm{p}+\mathrm{Au}, \mathrm{d}+\mathrm{Au}$ and ${ }^{3} \mathrm{He}+\mathrm{Au}$ collisions at $\sqrt{s_{N N}}=200 \mathrm{GeV}$ and beam energy scan down to $\sqrt{s_{N N}}=19.6 \mathrm{GeV}$ in $\mathrm{d}+\mathrm{Au}$ collisions.

PHENIX experiment has measured long range azimuthal correlation between various forward, central and backward detectors in order to extract the azimuthal event anisotropy which is possibly coming from the collectivity such as elliptic flow even in small systems at high multiplicity events. Fig. $[$ shows two particle correlation functions with 4 different combinations of detectors (CNT, FVTXS/N, BBCS/N) as indicated in the figure caption, where the magnitude of $2^{\text {nd }}$ order harmonic parameter of the fitted correlation increases with increasing the particle yield per unit $\eta$ (rapidity dependent freeze-out particle density, $d N / d \eta$ ) and with reducing the $\eta$-gap between the two detectors. Fig. $\square$ shows two particle correlation functions between FVTXS and FVTXN in $\mathrm{d}+\mathrm{Au}$ collisions at 4 different beam energies, 200, 62.4, 39 and $19.6 \mathrm{GeV}$, where the magnitude of $2^{\text {nd }}$ order harmonic parameter of the fitted correlation decreases with reducing the beam energy.

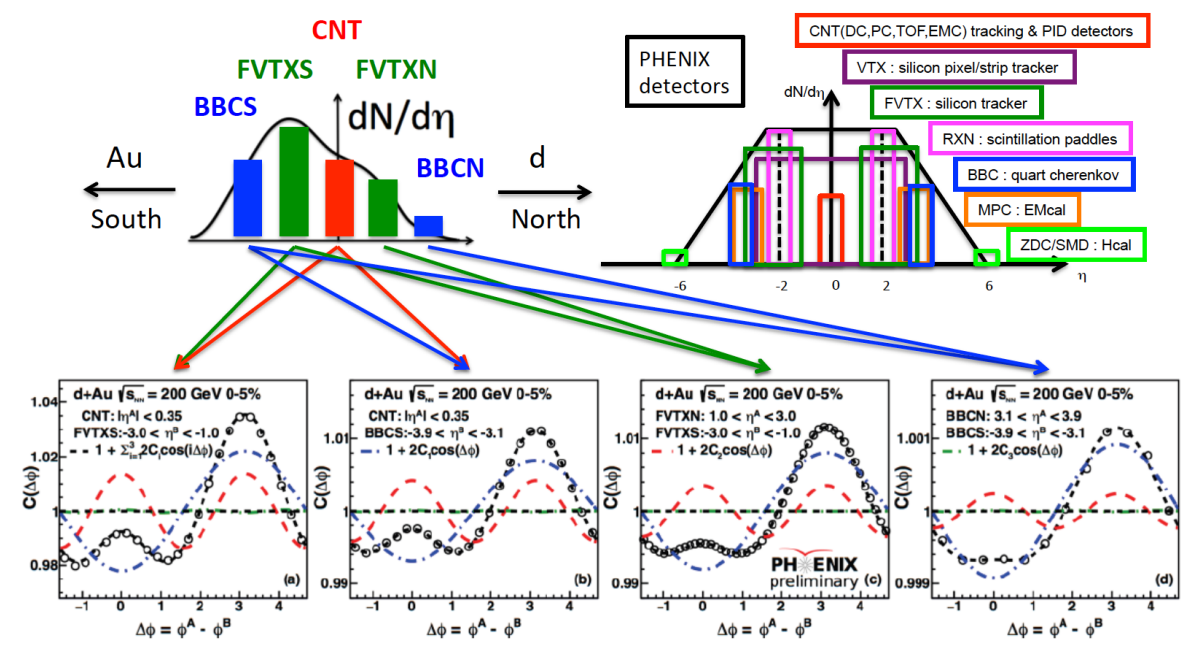

Figure 1: Two particle azimuthal correlation functions in $200 \mathrm{GeV} \mathrm{d}+\mathrm{Au}$ collisions are presented between various detector systems from forward, central and backward rapidity regions in PHENIX experiment with : (a) CNT - FVTXS, (b) CNT - BBCS, (c) FVTXN - FVTXS and (d) BBCN - BBCS, where CNT stands for central arm spectrometer $|\eta|<0.35, \operatorname{FVTX}(\mathrm{S}, \mathrm{N})$ stands for forward vertex detector $1<|\eta|<3, \mathrm{BBC}(\mathrm{S}, \mathrm{N})$ stands for beam beam counter $3.1<|\eta|<3.9$, and South and North $(\mathrm{S}, \mathrm{N})$ are for backward $(\eta<0)$ Au-going and forward $(\eta>0)$ d-going rapidities. [ए]] 


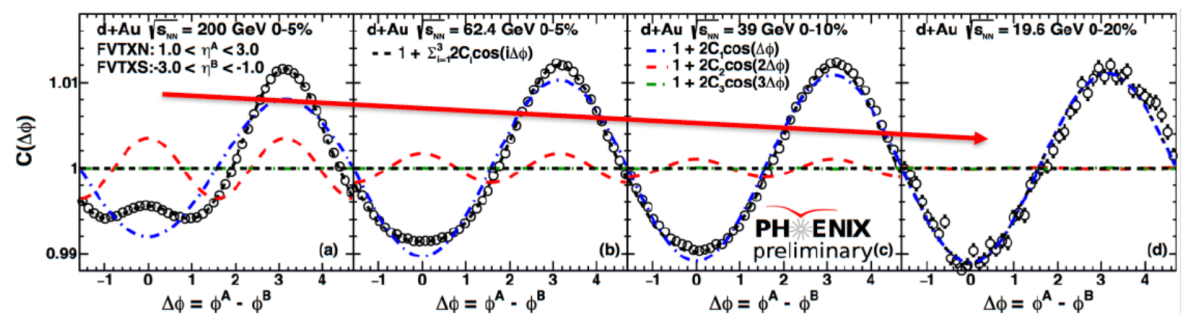

Figure 2: Two particle azimuthal correlation between FVTXS and FVTXN detectors in dAu collisions at (a) $200 \mathrm{GeV}$, (b) $62.4 \mathrm{GeV}$, (c) $39 \mathrm{GeV}$ and (d) $19.6 \mathrm{GeV}$. [ㅁ] ]

\section{Results from $\mathrm{p}+\mathrm{Au}, \mathrm{d}+\mathrm{Au}$ and ${ }^{3} \mathrm{He}+\mathrm{Au}$ collisions at $200 \mathrm{GeV}$}

In Fig. B1, the extracted $v_{2}$ parameters of charged particles at mid-rapidity $|\eta|<0.35$ are shown as a function of $p_{T}$ for 3 different colliding systems $\mathrm{p}+\mathrm{Au}, \mathrm{d}+\mathrm{Au}$ and ${ }^{3} \mathrm{He}+\mathrm{Au}$ at $\sqrt{s_{N N}}=200 \mathrm{GeV}$ for $0-5 \%$ central collisions, where the centrality is determined by the charged particle multiplicity in backward region at $-3.9<\eta<-3.1$ (BBCS). The sizable elliptic event anisotropy parameters $v_{2}$ of about $10-15 \%$ at $p_{T} 2-3 \mathrm{GeV} / \mathrm{c}$ are observed in all 3 systems and the results also show rather good agreement with some of the hydro-dynamic calculations.

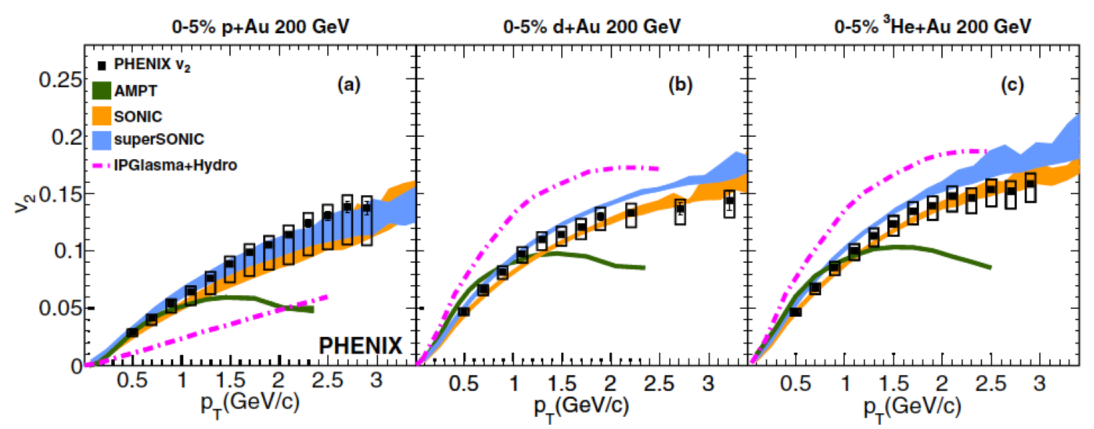

Figure 3: Charged particle $v_{2}$ parameters at mid-rapidity in central $\mathrm{p}+\mathrm{A}, \mathrm{d}+\mathrm{A}$ and ${ }^{3} \mathrm{He}+\mathrm{Au}$ collisions at $\sqrt{s_{N N}}=200 \mathrm{GeV}$ are shown as a function of $p_{T}$. Comparison with various hydro-dynamical and partonic cascade models are also shown for each colliding system. [[, \&, Q]

The extracted $v_{2}$ among 3 colliding systems are directly compared in Fig.⿴囗十 panel (a), and the participant eccentricity scaled $v_{2}$ based on Glauber simulation are compared in panel (b). The larger $v_{2}$ parameter has been observed for larger system size from $\mathrm{p}+\mathrm{Au}$ to ${ }^{3} \mathrm{He}+\mathrm{Au}$, however after scaling with the initial geometrical eccentricity coming from the participant fluctuation, $v_{2} / \varepsilon_{2}^{\text {Glauber }}$ becomes the largest for $\mathrm{p}+\mathrm{Au}$ collisions, which could be because the larger initial density is achieved with the smaller volume of participant for the same fraction (top $5 \%$ ) of centrality among 3 systems. Small but finite $v_{3}$ parameters are observed in central $\mathrm{d}+\mathrm{Au}$ and especially significant in central ${ }^{3} \mathrm{He}+\mathrm{Au}$ collisions at $200 \mathrm{GeV}$ as shown in panel (c) of Fig.G.

Event anisotropy $v_{2}$ of identified particles charged pions $\pi^{+}+\pi^{-}$and (anti-) protons $p+\bar{p}$ are measured in the mid-rapidity $|\eta|<0.35$ at $200 \mathrm{GeV} \mathrm{p}+\mathrm{Au}, \mathrm{d}+\mathrm{Au}$ and ${ }^{3} \mathrm{He}+\mathrm{Au}$ collisions as shown in Fig.5 panels (a), (b) and (c). The mass ordering which is usually interpreted as hydro-dynamical nature of elliptic and radial expansion in heavy-ion collisions are also observed here in these small 

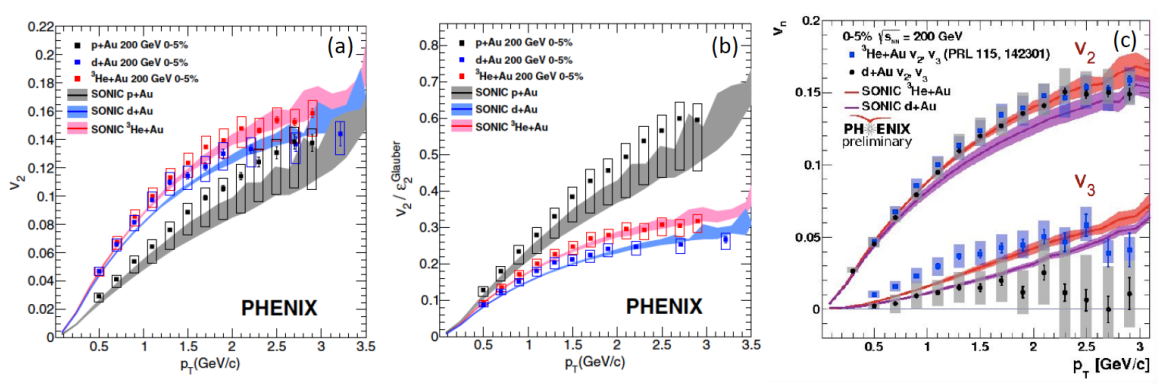

Figure 4: The comparison of $v_{2}$ and scaled $v_{2} / \varepsilon_{2}^{\text {Glauber }}$ are shown in panel (a) and (b) among 3 colliding systems p+Au, $\mathrm{d}+\mathrm{Au}$ and ${ }^{3} \mathrm{He}+\mathrm{Au}$. [四] $v_{2}$ and $v_{3}$ are compared in panel (c) in $\mathrm{d}+\mathrm{Au}$ and ${ }^{3} \mathrm{He}+\mathrm{Au}$ collisions.

collision systems, where the mass splitting seems to increase with the system size from $\mathrm{p}+\mathrm{Au}$ to $\mathrm{d}+\mathrm{Au}$ and ${ }^{3} \mathrm{He}+\mathrm{Au}$. While in the $p_{T}$ region above $1.5-2 \mathrm{GeV} / \mathrm{c}$, there seems to be reversed trend of $v_{2}$ magnitude between pions and (anti-) protons which can be interpreted as baryon and meson difference based on quark coalescence picture as a hadronization mechanism from the quark phase that is also similar to the measurements in heavy-ion collisions. Fig.\$ panels (d), (e) and (f) show the number of constituent quark scaled $v_{2}$ for 3 collision systems, where the agreement of scaled $v_{2}$ between pions and (anti-) protons are equally good for all 3 systems, but there could be a hint of better agreement in larger system $\left({ }^{3} \mathrm{He}+\mathrm{Au}\right)$ than in smaller system $(\mathrm{p}+\mathrm{Au})$.
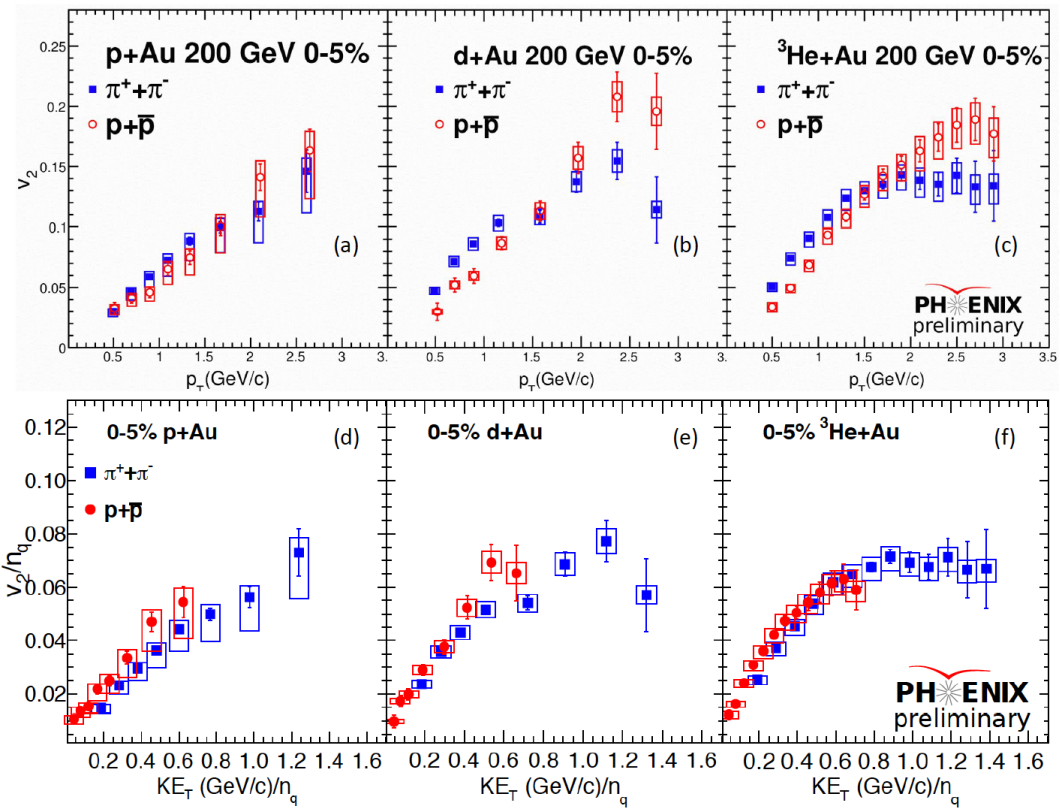

Figure 5: The identified particles $v_{2}$ parameters are shown for $\pi^{+}+\pi^{-}$and $p+\bar{p}$ as a function of $p_{T}$ in central $0-5 \% \mathrm{p}+\mathrm{Au}, \mathrm{d}+\mathrm{Au}$ and ${ }^{3} \mathrm{He}+\mathrm{Au}$ collisions at mid-rapidity $|\eta|<0.35$ in upper panels. The number of quark scaled $v_{2}$ parameters are shown as a function of scaled kinetic energy $\left(m_{T}-m_{0}\right) / n_{\text {quark }}$ in lower panels. [एत]

The identified particles $v_{2}$ parameters are compared with model calculations in Fig.6, the upper panels (a), (b) and (c) are with hydro-dynamical model (superSONIC) and the lower panels (d), (e) and (f) are with transport model (AMPT including partonic and hadronic cascades). The both 
models describe the data at lower $p_{T}$ region rather well especially on the mass dependence of $v_{2}$ parameters between $\pi^{+}+\pi^{-}$and $p+\bar{p}$. The qualitative feature of reversed trend of $v_{2}$ between $\pi^{+}+\pi^{-}$and $p+\bar{p}$ at higher $p_{T}$ region is also seen in both models, however the detailed dependence of the trend (magnitude of the difference as well as $p_{T}$ dependent shapes) are quite different from the experimental data.

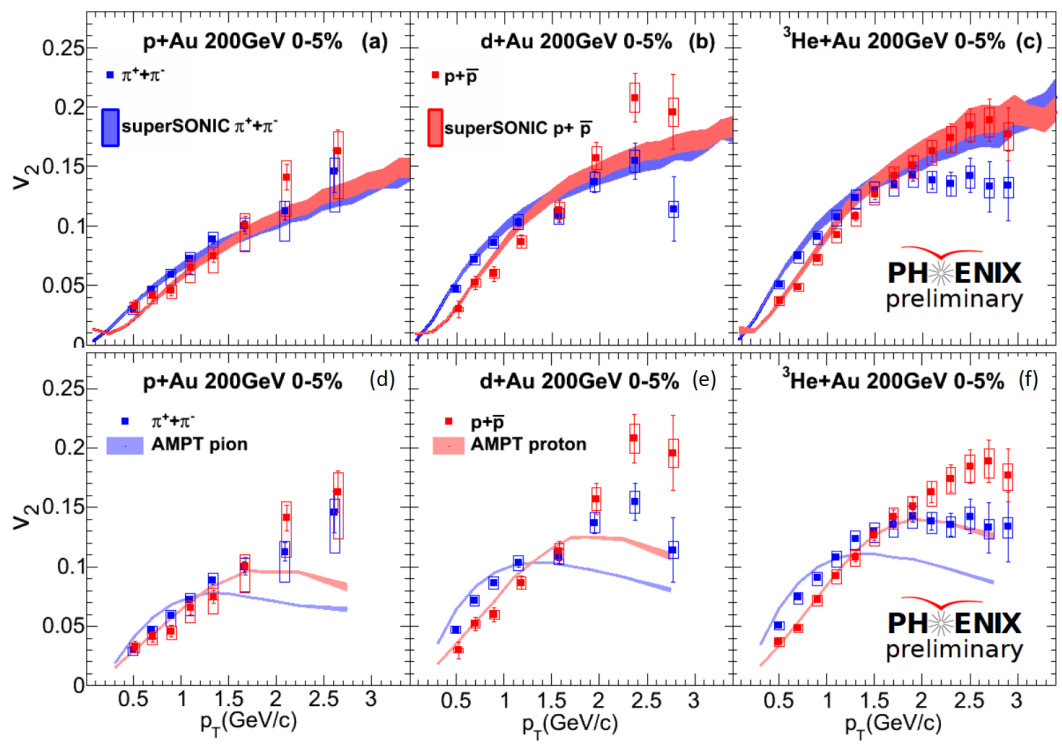

Figure 6: The identified particles $v_{2}$ parameters $\pi^{+}+\pi^{-}$and $p+\bar{p}$ are compared with hydro-dynamical model superSONIC in upper panels and with transport model AMPT in lower panels. [ए]]

\section{Results from d+Au collisions at 19.6, 39, 62.4 and $200 \mathrm{GeV}$}

The charged particle $v_{2}$ parameters are measured at mid-rapidity $|\eta|<0.35$ in central d+Au collisions for 4 different beam energies, $200 \mathrm{GeV}$ as shown in Fig.ర panel (a), $62.4 \mathrm{GeV}$ in panel (b), $39 \mathrm{GeV}$ in panel (c) and $19.6 \mathrm{GeV}$ in panel (d). In the $p_{T}$ region below $1 \mathrm{GeV} / \mathrm{c}$, the measured $v_{2}$ parameters have been found to be all similar among different beam energies, which does not mean $\left\langle v_{2}\right\rangle$ is unchanged in these energy region, since $\left\langle p_{T}\right\rangle$ is known to vary. However at higher $p_{T}$ region above $1.5-2 \mathrm{GeV} / \mathrm{c}$, the measured $v_{2}$ parameters have been observed to increase with reducing the beam energy especially at 39 and $19.6 \mathrm{GeV}$, which seems to be caused by an increasing non-flow contribution, especially because the relative non-flow correlation increases with the reduction of multiplicity at lower beam energy. The upper panels (a), (b), (c) and (d) of Fig.D are compared with hydro-dynamic models SONIC/superSONIC without/with pre-flow field in the model, where no significant but slightly increasing $v_{2}$ magnitude is seen in the model calculation with increasing the beam energy and one should note that the model calculation agrees with the experimental data very well at 62.4 and $200 \mathrm{GeV}$ central d+Au collisions. The lower panels (e), (f), $(\mathrm{g})$ and $(\mathrm{h})$ of Fig. $\square$ are compared with AMPT model using two different $v_{2}$ definitions, one $v_{2}\{\mathrm{EP}\}$ defined with respect to the Event Plane, which can also be done in experimental data analysis using the freeze-out hadrons, another $v_{2}\{\mathrm{PP}\}$ defined with respect to the initial parton plane, which can 
be calculated with initial geometry of partons in participants. While $v_{2}\{\mathrm{PP}\}$ is mostly independent with beam energy, but $v_{2}\{\mathrm{EP}\}$ seems to catch the trend of the experimental data, that could be explained by the non-flow contribution as mentioned above.

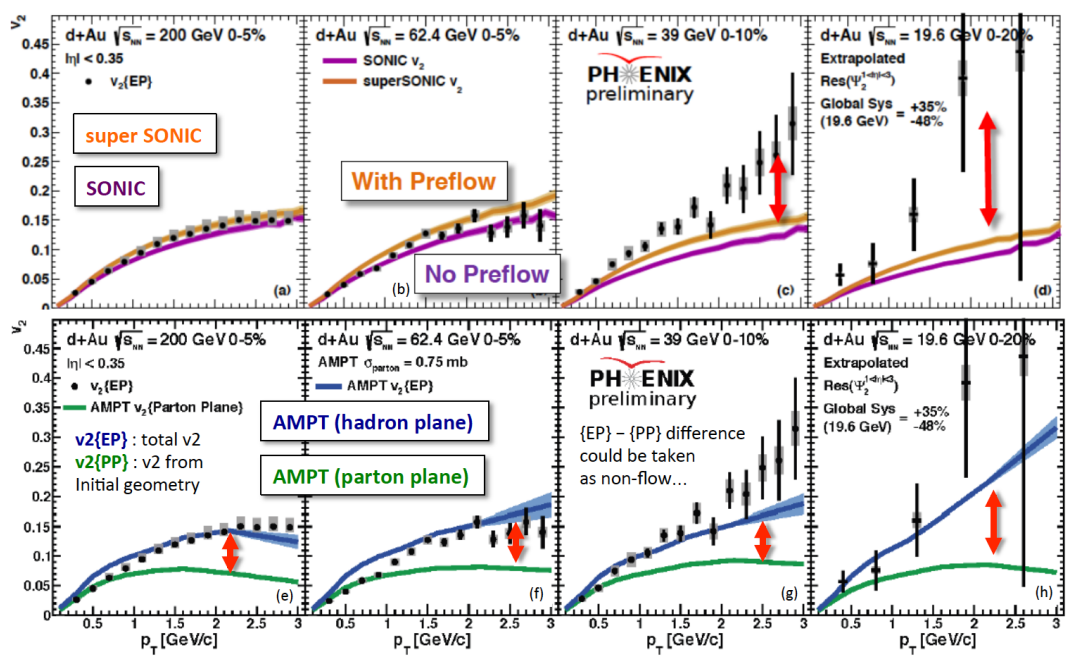

Figure 7: The charged particle $v_{2}$ parameters at mid-rapidity $|\eta|<0.35$ in central $\mathrm{d}+\mathrm{Au}$ collisions are shown for 4 different beam energies, 200, 62.4, 39 and $19.6 \mathrm{GeV}$. [U]] The experimental data points are the same between upper and lower panels, while the different model calculations (hydro-dynamic model SONIC/superSONIC in upper panels and transport model AMPT in lower panels) are compared with data.

The rapidity dependence of the charged particle $v_{2}$ parameters are measured with central, forward and backward detectors between -3 and 3 in $\eta$ in central $\mathrm{d}+\mathrm{Au}$ collisions for 4 different beam energies, as shown in Fig. 18 panels (a)-(d). One can note that $v_{2}$ at mid-rapidity does not show very strong energy dependence, as also discussed with previous Fig.Z, however the shape of rapidity dependence is quite different between the different beam energies and rather significant forward and backward asymmetry has been observed. The measured $v_{2}$ in the backward rapidity region shows very strong beam energy dependence, where the magnitude of $v_{2}$ around $\eta \sim-2$ decreases with beam energy quite a lot and could even change the sign at the lowest beam energy of $19.6 \mathrm{GeV}$. AMPT model calculation can generally describe the experimental measurements in forward and central rapidity regions, but fails to explain especially in backward rapidity region.
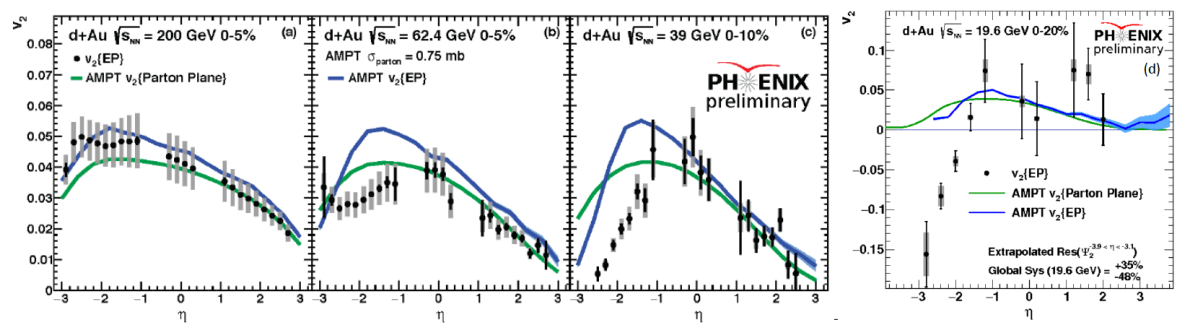

Figure 8: The rapidity dependence of the charged particle $v_{2}$ parameters are shown in central d+Au collisions for 4 different beam energies, 200, 62.4, 39 and $19.6 \mathrm{GeV}$. [U]] AMPT model calculations are compared with data. 
The multi-particle cumulant analysis is applied using forward and backward regions $1<|\eta|<3$ as shown in Fig. 9 . As seen in $\mathrm{p}+\mathrm{Pb}$ collisions at LHC energy, the real values of $v_{2}\{4\}$ has also been observed in $\mathrm{d}+\mathrm{Au}$ collisions at RHIC energy down to $19.6 \mathrm{GeV}$, while the complex values of $v_{2}\{4\}$ in $\mathrm{p}+\mathrm{p}$ collisions at LHC or $\mathrm{p}+\mathrm{Au}$ collisions at RHIC are seen in contrast. The measured $v_{2}\{2\}$ has been found to be quite different from $v_{2}\{2,|\Delta \eta|>2\}$, because of rather different kinematic conditions especially in terms of short-range small $\Delta \eta$ correlation as well as weighting between forward and backward rapidities. The measured $v_{2}\{4\}$ has been found to increase with reducing the beam energy, which seems to be reproduced by AMPT model calculation. [ए2]

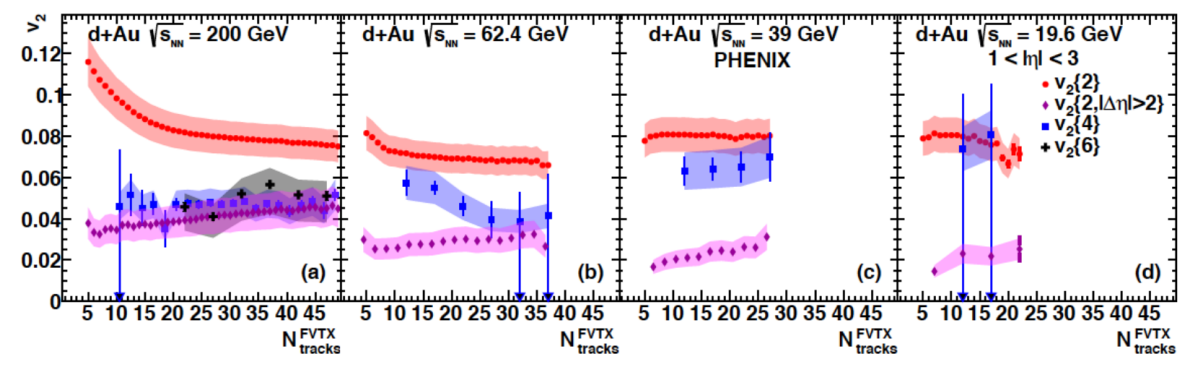

Figure 9: The multi-particle cumulant results $v_{2}\{2\}, v_{2}\{4\}$ and $v_{2}\{6\}$ in $\mathrm{d}+\mathrm{Au}$ collisions as a function of measured multiplicity in forward and backward rapidity for 4 different beam energies, 200, 62.4, 39 and $19.6 \mathrm{GeV}$, compared with $v_{2}\{2,|\Delta \eta|>2\}$. [ए]]

\section{Reference fitting method to see a small flow signal}

In order to extract a small flow-like signal from the azimuthal correlation that includes large non-flow background, the reference fitting method is proposed in a data driven way using AMPT simulation at $500 \mathrm{GeV}$ p $+\mathrm{p}$ collisions as an example in Fig. $\mathrm{W}$. The reference function $f(x)$ can firstly be determined by a correlation function $C_{2}$ from low multiplicity data sample or by minimum bias data sample. The multiplicity (or centrality) dependent correlation functions $C_{2}$ can then be fitted with the reference function $F(x)=A+B f(x)$ with two additional free parameters $A$ and $B$. The ratio of correlation function $C_{2}$ over the fitted function $F(x)$ (or subtracted correlation function $\left.1+C_{2}-F(x)\right)$ can finally be looked at for further analysis in order to look for any possible change of correlation shape as a function of multiplicity or centrality. This method is similar to the template fitting method used in ATLAS Experiment at LHC [], however the spirit how one defines the final anisotropy is quite different as mentioned here. This method should reveal any small change in the measured correlation function that could be given by a possible collective and elliptic expansion in small system or by any small modification of jet-like signal, which can not fully be separated experimentally.

\section{Summary}

Recent results on flow in small systems from PHENIX experiment are presented. System size dependence of small collision systems $\mathrm{p}+\mathrm{Au}, \mathrm{d}+\mathrm{Au}$ and ${ }^{3} \mathrm{He}+\mathrm{Au}$ collisions at $200 \mathrm{GeV}$ including particle species dependence and beam energy dependence from 20 to $200 \mathrm{GeV} \mathrm{d}+\mathrm{Au}$ collisions are 


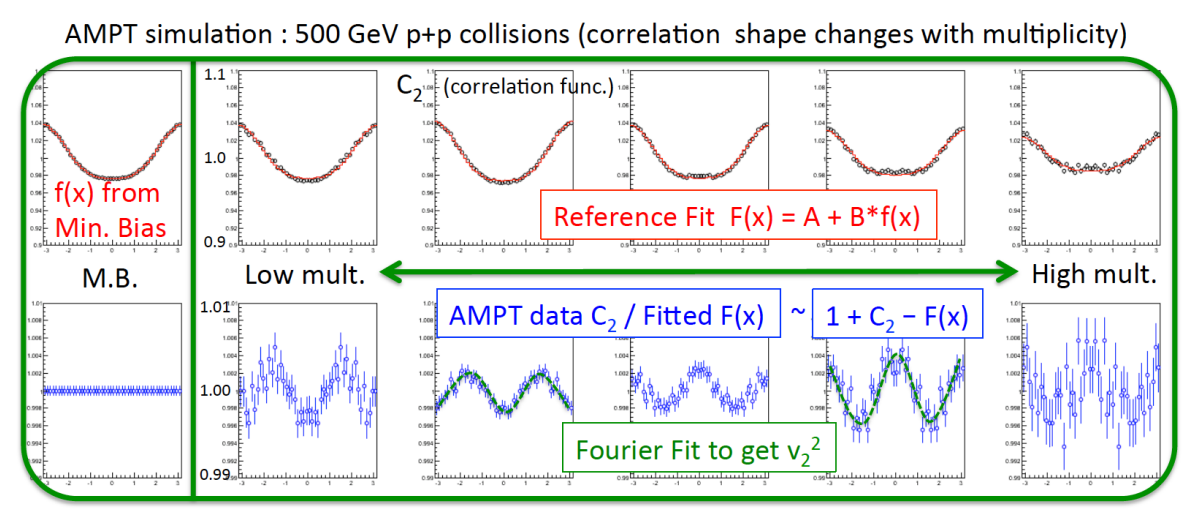

Figure 10: AMPT simulation at $500 \mathrm{GeV} p+p$ collisions is used to test a proposed reference fitting method in order to observe a small change in correlation functional shape as a function of multiplicity.

discussed. The extracted elliptic event anisotropy parameter $v_{2}$ can be interpreted as a signal of collective and elliptic expansion, which is originally driven by the initially small and anisotropic geometry of fluctuating participant density distribution. A significant change in rapidity dependence of elliptic anisotropy parameter $v_{2}$ are observed with different beam energies, which would naturally include significant contributions from spectator at low beam energies especially in backward rapidity region. The full interpretation of these results would require more complete centrality and rapidity dependent studies yet to come in near future. A new method to extract any small flow signal has been proposed and discussed.

\section{References}

[1] V. Khachatryan et al. (CMS Collaboration), J. High Energy Phys. 09, 091 (2010)

[2] G. Aad et al. (ATLAS Collaboration), Phys. Rev. Lett. 116, 172301 (2016)

[3] V. Khachatryan et al. (CMS Collaboration), Phys. Rev. Lett. 116, 172302 (2016)

[4] S. Chatrchyan et al. (CMS Collaboration), Phys. Lett. B718, 795 (2013)

[5] B. Abelev et al. (ALICE Collaboration), Phys. Lett. B719, 29 (2013)

[6] G. Aad et al. (ATLAS Collaboration), Phys. Rev. Lett. 110, 182302 (2013)

[7] C. Aidala et al. (PHENIX Collaboration), Phys. Rev. C95, 034910 (2017)

[8] A. Adare et al. (PHENIX Collaboration), Phys. Rev. Lett. 114, 192301 (2015)

[9] A. Adare et al. (PHENIX Collaboration), Phys. Rev. Lett. 115, 142301 (2015)

[10] A. Adare et al. (PHENIX Collaboration), arXiv:1710.09736

[11] C. Aidala et al. (PHENIX Collaboration), arXiv:1708.06983

[12] C. Aidala et al. (PHENIX Collaboration), arXiv:1707.06108 